(2) Open Access Full Text Article

\title{
Investigation of SARS-CoV-2 on Ocular Surface of Coronavirus Disease 2019 Patients Using One-Step Reverse-Transcription Droplet Digital PCR
}

\author{
Xian Zhang ${ }^{\mathrm{l}, *}$ \\ Liting Chen ${ }^{2 * *}$ \\ Gaoxiang Wang ${ }^{2, *}$ \\ Liwen Chen' \\ Lifang Huang ${ }^{2}$ \\ Yang $\mathrm{Cao}^{2}$ \\ Xing Chen ${ }^{2}$ \\ Chaohua Deng' \\ Xuhui Chen' \\ Dandan Ke' \\ Yuanjun Qin' \\ Bo Chen' \\ Xufang Sun' \\ 'Department of Ophthalmology, Tongji \\ Hospital, Tongii Medical College, \\ Huazhong University of Science and \\ Technology, Wuhan, People's Republic of \\ China; ${ }^{2}$ Department of Hematology, \\ Tongji Hospital, Tongji Medical College, \\ Huazhong University of Science and \\ Technology, Wuhan, People's Republic of \\ China
}

*These authors contributed equally to this work
Purpose: This study detects SARS-CoV-2 in the ocular surface through one-step reversetranscription droplet digital PCR (one-step RT-ddPCR) and evaluates the possibility of the ocular surface as a possible transmission route.

Methods: A single-center prospective observational study was designed to investigate the viral loads in ocular surface. Specimens including the conjunctival swabs, nasopharyngeal swabs and blood were synchronously collected at a single time point for all COVID-19 patients. SARS-CoV-2 loads in nasopharyngeal swabs were tested by real-time polymerase chain reaction (PCR); the blood samples and conjunctival swabs were tested by real-time PCR and one-step RT-ddPCR.

Results: Sixty-eight COVID-19 patients confirmed by nasopharyngeal real-time PCR were recruited. In the single time point test, 40 cases showed positive SARS-CoV-2 detection in either the blood, tears, or nasopharynx, of which four cases were triple-positive, 10 were dual-positive, and 26 were single-positive. The positive rate of nasopharyngeal swab realtime PCR test was $22.1 \%$ (15/68). The positive rate of blood and conjunctival swabs by onestep RT-ddPCR was 38.2\% (26/68) and 25\% (17/68), respectively, whereas real-time PCR was all negative. Positive conjunctival swabs were significantly correlated with positive nasopharyngeal swabs $(P=0.028)$. The sampling lags from illness onset to sampling day in 3 out of 4 triple-positive patients and in 9 out of 10 dual-positive patients were respectively less than 9 days and less than 20 days.

Conclusion: Our results indicate that the positive rate of SARS-CoV-2 on the ocular surface is much higher than expected. Transmission possibility through the ocular surface may be greatly underestimated.

Keywords: absolute quantification, virus detection, transmission, nasopharyngeal specimens, blood samples, tear samples

\section{Introduction}

Coronavirus disease 2019 (COVID-19) is a global pandemic and as of November 3, 2020, nearly 46 million cases and 1.2 million deaths have been reported globally. ${ }^{1}$ The highly contagious COVID-19 is caused by the infection of the SARS-CoV-2 virus, and respiratory tract transmission and contact transmission are considered its main infection routes. ${ }^{2,3}$ The SARS-CoV-2 virus has been tested in nasopharyngeal swabs as well as in other specimens such as feces, ocular secretions, and blood, suggesting that various potential transmission routes of this previously unknown infectious virus should be considered seriously. ${ }^{4,5}$
Correspondence: Bo Chen; Xufang Sun Department of Ophthalmology, Tongj Hospital, Tongii Medical College,

Huazhong University of Science and Technology, 1095 jiefang Ave., Wuhan, 430030, People's Republic of China Email eyechen@aliyun.com; sunxufang2016@I63.com 
Whether the ocular surface can transmit the virus has been widely debated. A previous study reported that realtime polymerase chain reaction (PCR) failed to detect SARS$\mathrm{CoV}-2$ in the tears of COVID-19 patients; $^{6}$ other groups have detected SARS-CoV-2 nucleic acids in the tears of very a few COVID-19 patients by using SARS-CoV-2 real-time PCR assays. $^{7-9}$ Thus, the overall positive rate of conjunctival swabs by real-time PCR are extremely low. However, considering that real-time PCR has a non-negligible falsenegative rate even in the pharynx tests and the sample volume of tears is far less than the pharynx sample, the falsenegative rate for SARS-CoV-2 real-time PCR tests for tears should be seriously excluded. In other words, more sensitive methods are needed to determine whether there is SARSCoV-2 in ocular surface.

Droplet digital PCR (ddPCR) is an absolute nucleic acid quantification method that affords many advantages over real-time PCR for virus detection, including the ability to obtain absolute quantification without external references and robustness to variations in PCR efficiency. ${ }^{10}$ Several studies showed that ddPCR is more sensitive and reliable for detecting low-viral-load samples than real-time PCR in COVID-19 patients. ${ }^{10-12}$ Similarly in our latest study, one-step RT-ddPCR was proved to be a highly sensitive detection method with a $3.03 \%$ of false positive rate and lower $50 \%$ confidence interval of detection (LOD50) at 54.026 copies/mL plasma. ${ }^{13}$

In this study, considering the sensitivity limitations of real-time PCR and the low sample volume of tears, we applied one-step reverse-transcription ddPCR (RT-ddPCR) for conjunctival swabs, which is even more sensitive than conventional ddPCR to determine the viral load of SARSCoV-2 on ocular surface.

\section{Methods}

\section{Study Design and Participants}

Participants presented in this single-center prospective observational study were hospitalized newly diagnosed COVID19 pneumonia patients $(\mathrm{N}=68)$ with informed consents between February 12, 2020 and March 10, 2020, in Tongji Hospital of Huazhong University of Science and Technology in Wuhan, China, according to the WHO guidance. ${ }^{14}$ The diagnosis and the illness severity of COVID-19 were defined according to the Chinese management guideline for COVID19 (version 7.0) and the World Health Organization interim guidance. ${ }^{14}$ Patients meeting any of the following criteria were defined as severe status: 1$)$ respiratory distress $(\geqq 30$ breaths/min); 2) oxygen saturation $\leq 93 \%$ at rest; 3 ) arterial partial pressure of oxygen $\left(\mathrm{PaO}_{2}\right)$ /fraction of inspired oxygen $\left(\mathrm{FiO}_{2}\right) \leqq 300 \mathrm{mmHg}(1 \mathrm{mmHg}=0.133 \mathrm{kPa})$. This study complied with the Declaration of Helsinki and was approved by the Ethics Committee of Tongji Hospital (TJ-C20200113) - Informed consent was obtained from each patient or patients' relatives if patients were not in good condition. Exclusion criteria of this study enrollment included: 1) suspicious cases without positive nasopharyngeal swab results by RT-PCR; 2) no written informed consent could be obtained; 3) patients had received remdesivir or were involved in other clinical trials.

\section{Specimens and Clinical Data Collection}

Every participant synchronously provided three types of specimens including serum, nasopharyngeal swabs and conjunctival swabs on the enrollment day. All samples were collected and immediately transferred to a $4^{\circ} \mathrm{C}$ refrigerator and processed within 24 hours. Harvested serum and viral-transport medium for swab preservation were immediately assayed or aliquoted to be stored at $\leq-80$ ${ }^{\circ} \mathrm{C}$. Repeated freeze-thaw cycles were avoided. All types of these samples were tested by the well-accepted conventional RT-PCR. In light of the relative lower SARS-CoV-2 detection rate of serum and tear specimens by RT-PCR, these two types of samples were additionally detected by one-step RT-ddPCR. Clinical informations were inputted in an electronic data capture system (EDCS) and extracted for further analysis. The time from the illness onset to specimens' collection was defined as the sampling lag.

\section{Detection of SARS-CoV-2 by Conventional Real-Time PCR}

SARS-CoV-2 was tested through the real-time PCR of SARSCoV-2 RNA, as reported previously. ${ }^{15}$ Two target genes, including open reading frame $1 \mathrm{ab}$ (ORF1ab) and nucleocapsid protein $(\mathrm{N})$, were simultaneously amplified and tested during the real-time PCR assay (Supplementary Table S1).

A cycle threshold value (Ct-value) less than 37 was defined as a positive test, and a Ct-value of 40 or more was defined as a negative test. These diagnostic criteria were based on the recommendation by the National CDC (China). A medium load, defined as a $\mathrm{Ct}$ value of 37 to less than 40 , required confirmation by at least two repeats. Samples were considered as negative when their $\mathrm{Ct}$ values were higher than 40 . 


\section{Plasmid Standards Preparation}

SARS-CoV-2 plasmids with orflab inserted (Sangon Biotech, Shanghai, China) were linearized by restriction enzyme BamHI, serially diluted and subjected to ddPCR amplification.

\section{Detection of SARS-CoV-2 by One-Step RT-ddPCR and Viral Load Calculation}

Detailed protocol and quality control of one-step RTddPCR of SARS-CoV-2 RNA was referred to our recent published article. ${ }^{13}$ Briefly, for the quantitative detection of SARS-CoV-2 copy numbers, a viral RNA purification kit (QIAamp Viral RNA Mini Kit, Qiagen 52904\#, Germany), one-step RT-ddPCR advanced kit, QX200 droplet generator (BioRad, USA), and QX200 droplet reader (BioRad, USA) were used following the manufacturers' instructions. A 2-well test was performed for each sample in this study. The SARS-CoV-2 specific minor groove binder (MGB) probe-primer set was designed for targeting the orflab region, and the sequences were attached in Supplementary Table S1. The probe-primer set has been previously reported by our center. ${ }^{13}$

For ddPCR analysis, QuantaSoft software version 1.7.4 (Bio-Rad, USA) was used to quantify the copies per microliter of the target per well. Thresholds were determined manually for each experiment according to negative controls that included a no-template control and four healthy samples. Droplet positivity was determined by the fluorescence intensity; only droplets above a minimum amplitude threshold were counted as positive. Negative control plasma and conjunctival samples from 16 healthy persons were used to estimate the specificity of the probe. LLoD which is defined as the lowest concentration at which $50 \%$ of positive samples were detected using serially diluted plasmid standards in eight replicates. All clinical samples were run in duplicate, unless otherwise specified, and the viral load was taken as the average of the two measurements.

\section{Statistical Analysis}

Categorical variables were described using the frequency rates and percentages, and continuous variables were described using the median and interquartile range (IQR). Univariate analyses were assessed with $\chi 2$ test of association for categorical values, and $P<0.05$ was considered a significant difference. Multiple comparisons were made by utilizing Dunn's multiple comparisons test. Probit analysis for lower limit of detection (LLoD) was conducted with GraphPad Prism 7.0.

\section{Results \\ Basic Clinical Characteristics of Enrolled Patients}

Of these 68 enrolled COVID-19 patients, none of them had any symptoms regarding viral conjunctivitis. The median age was 58.3 years (IQR: 47-70) with gender ratio of 33/35 (M/F). The general and severe status was $81.9 \%$ and $19.1 \%$, respectively. Of note, the study cohort did not contain critical status since most of these patients could not afford written informed consents. Comorbidities were present in $48.5 \%$ of the patients, with hypertension and diabetes being the most common ones. Sampling lags were ranging from 6 days to over 1 month. Fourteen of $68(20.6 \%)$ patients provided samples less than 10 days from illness onset, whereas 20 of which $(29.4 \%)$ was over 20 days. The majority (50\%) of sampling lags was ranging from 10 to 20 days after the onset time. According to CT scans, 53 patients showed bilateral pneumonia (77.9\%) and just 15 (22.1\%) showed unilateral pneumonia (Table 1).

\section{The Performance of ddPCR in Detecting SARS-CoV-2 Viral Loads}

Commercial SARS-CoV-2 plasmids were used to evaluate the accuracy and LLoD of ddPCR detection. Plasmids were

Table I Basic Clinical Characteristics of Enrolled Patients

\begin{tabular}{|l|l|}
\hline Study Population & $\mathbf{N}(\%)$ \\
\hline No. of patients & 68 \\
Age, median (IQR), y & $58.3(47-70)$ \\
Gender & \\
Male & $33(48.5)$ \\
Female & $35(5.5)$ \\
Severity & \\
General & $55(81.9)$ \\
Severe & $13(19.1)$ \\
Sampling lags & \\
$<$ I0 days & $14(20.6)$ \\
I0-20 days & $34(50)$ \\
$>20$ days & $20(29.4)$ \\
Comorbidities & $33(48.5)$ \\
Hypertension & $25(36.8)$ \\
Diabetes & $12(17.6)$ \\
Coronary heart disease & $3(4.4)$ \\
CT Laterality & \\
Unilateral & $15(22.1)$ \\
Bilateral & $53(77.9)$ \\
\hline
\end{tabular}

Note: ${ }^{\text {a }}$ The time from illness onset to the conjunctival sampling. 
twofold serially diluted at the concentrations ranging from approximately 800 copies $/ \mu \mathrm{L}$ to 12.5 copies $/ \mu \mathrm{L}$ and subjected to ddPCR amplification. Each concentration, measured in triplicate, was well in line with expected concentration $\left(\mathrm{R}^{2}=\right.$ $0.998, P<0.001$ ), indicating that ddPCR using the probeprimer set for SARS-CoV-2 could accurately quantify SARSCoV-2 copies (Figure 1A). To determine the LLoD which is defined as the lowest concentration at which $50 \%$ of positive samples were detected, eight replicates of each concentration were tested. The LLoD of plasmid standards was 10 copies per reaction (Figure 1B). The probe-primer set showed high specificity as all negative control samples had zero viral load. Thus, the false positivity for this probe-primer set was 0 .

\section{Very Low SARS-CoV-2 Loads Were Detected in Both Blood and Tears of COVID-I 9 Patients}

We tracked the presence of the SARS-CoV-2 RNA in tear and blood samples from 68 patients using real-time PCR and one-step RT-ddPCR. Notably, viral load in our cohort was not detected either in blood or tears using real-time PCR. On the contrary, SARS-CoV-2 RNA was detected in both blood and tear by one-step RT-ddPCR, and the positive rates were $38.2 \%(26 / 68)$ and $25 \%(17 / 68)$, respectively. However, the viral loads in these positive cases were generally very low, ranging from 125 to 553.6 copies $/ \mathrm{mL}$ (Figure 2), which below the detection limit of real-time PCR.

\section{Distribution Features of SARS-CoV-2 in the Blood, Tears, and Nasopharyngeal Samples During COVID-19 Pneumonia Courses}

The positive rate of nasopharyngeal swabs in our single time point test was only $22.1 \%$ (15/68). In total, 40 cases

A

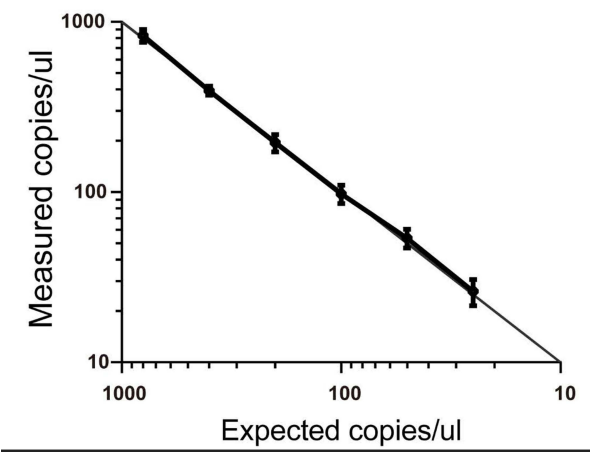

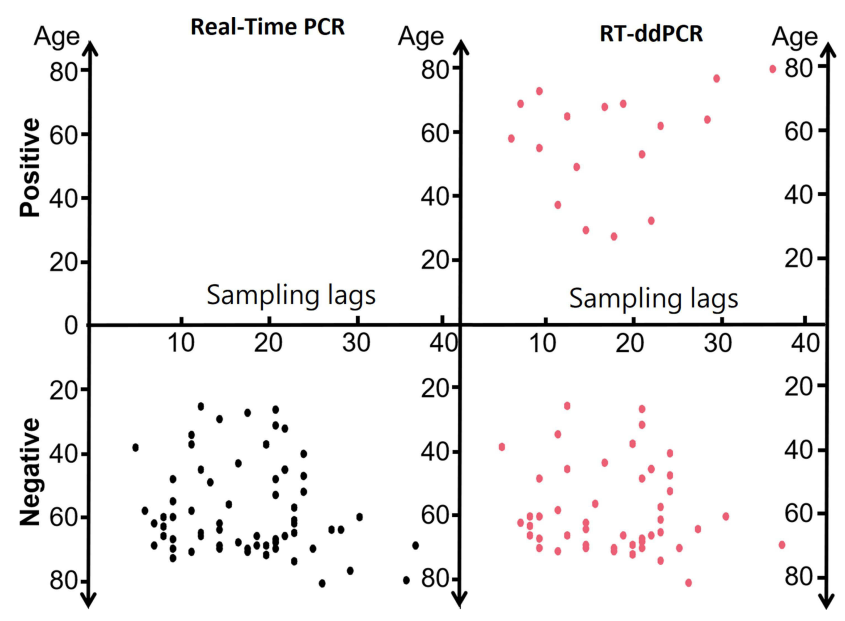

Figure 2 Comparison of positive rates of SARS-CoV-2 detection in tears using different methods.

Abbreviations: Real-time PCR, real-time polymerase chain reaction; RT-ddPCR, reverse-transcription droplet digital polymerase chain reaction; sampling lags, the time from illness onset to the conjunctival sampling.

showed positive SARS-CoV-2 detection in either the blood, tears, or nasopharynx, of which four cases were triple-positive, 10 were dual-positive; and 26 were singlepositive (Figure 3). Moreover, the sampling lags from illness onset to sampling day in 3 out of 4 triple-positive patients and in 9 out of 10 dual-positive patients were respectively less than 9 days and less than 20 days, indicating that the multiple-positive patients are mainly identified at a relatively early stage of COVID-19 (Figure 3).

\section{Positive One-Step RT-ddPCR Tear Specimens are Significantly Correlated with Positive Real-Time PCR Nasopharyngeal Specimens}

Table 2 shows the factors associated with the positive rate for one-step RT-ddPCR. Clinical factors were compared

\section{B}

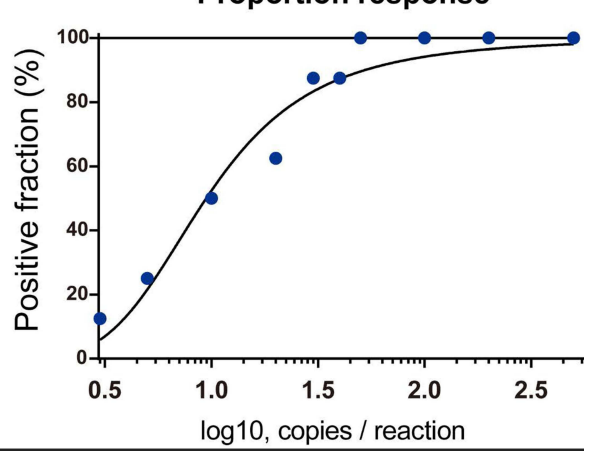

Figure I Evaluation of SARS-CoV-2 probe-primer set using plasmid standards. (A) Dilution curve of plasmid standards quantification by ddPCR. Correlation between expected and observed copy number were showed. Each black square represents a single replicate well of the dilution experiment, whereas the regression line is based on the average concentration at each dilution. (B) Probit analysis sigmoid curve reporting the lower limit of detection of ddPCR per reaction. 


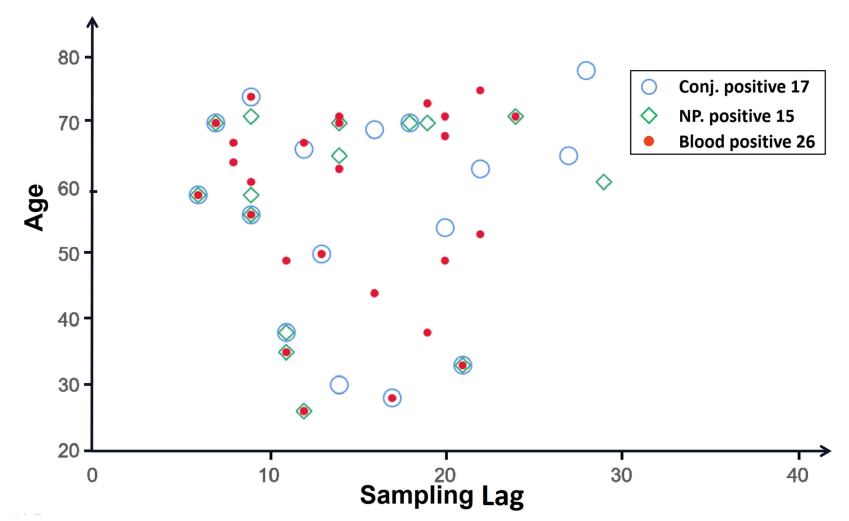

Figure 3 Distribution features of SARS-CoV-2 in the blood, tears, and nasopharyngeal samples during COVID-19 pneumonia courses.

Abbreviations: Conj, conjunctival; NP, nasopharyngeal.

between conjunctival viral positive and negative patients for one-step RT-ddPCR in terms of gender, severity of COVID-19, time from onset to sampling, CT laterality, and positive rate of blood. No significant statistical differences in these factors were found between these two groups. Positive conjunctival swabs seemed to be more likely to occur in elderly patients, but there was no statistical difference $(P=0.22$, Table 2$)$. Nevertheless, the SARS-CoV-2 viral loads of nasopharyngeal specimens were significantly correlated with those of conjunctival specimens $(P=0.028$, Table 2$)$.

\section{Discussion}

We systematically tested the viral RNA of SARS-CoV-2 in tear specimens by using real-time PCR and one-step RTddPCR. Our results indicate that the viral load in tears is very low, which is below the detection limit of real-time PCR, whereas one-step RT-ddPCR is more sensitive and yields a positive rate of $\sim 25 \%$ for the same tear samples. Taking account that the simultaneously positive rate of nasopharyngeal sample test in these previous positive cases was only $22.1 \%$, we speculate that the viral test by one-step RT-ddPCR in tears may yield a much higher positive rate if the test is performed earlier. Thus, the ability of transmission through the ocular surface may be greatly underestimated. When we are directly or indirectly exposed to patient's eye discharges or tears, appropriate ocular hygiene habits may be essential to prevent the spread of SARS-CoV-2. It is recommended for

Table 2 Correlation Analysis of Positive One-Step RT-ddPCR Tear Specimens

\begin{tabular}{|c|c|c|c|}
\hline Study Population (No.) & $\begin{array}{l}\text { Conjunctival } \\
\text { Positive }\end{array}$ & $\begin{array}{l}\text { Conjunctival } \\
\text { Negative }\end{array}$ & P-value \\
\hline No. of patients & 17 & 51 & \\
\hline Gender & & & 0.57 \\
\hline Male & 9 & 23 & \\
\hline Female & 8 & 28 & \\
\hline Severity & & & 0.85 \\
\hline General & 12 & 36 & \\
\hline Severe & 5 & 15 & \\
\hline Age & & & 0.22 \\
\hline$<50$ years & 4 & 15 & \\
\hline$>50$ years & 13 & 36 & \\
\hline Sampling lags & & & 0.81 \\
\hline$<10$ days & 4 & 9 & \\
\hline $10-20$ days & 7 & 20 & \\
\hline$>20$ days & 6 & 22 & \\
\hline CT Laterality & & & 0.61 \\
\hline Unilateral & 3 & 12 & \\
\hline Bilateral & 14 & 39 & \\
\hline Blood ddPCR ${ }^{a}$ & & & 0.77 \\
\hline Positive & 7 & 19 & \\
\hline Negative & 10 & 32 & \\
\hline Nasopharyngeal qPCR & & & $0.028^{*}$ \\
\hline Positive & 7 & 8 & \\
\hline Negative & 10 & 43 & \\
\hline
\end{tabular}

Notes: ${ }^{a}$ Samples acquired from the same day of conjunctival sampling; ${ }^{* P}<0.05$. 
clinicians and ophthalmologists to use personal protective equipment (PPE) for eyes while examining patients.

Although nasopharyngeal swab tests for SARS-CoV-2 RNA by real-time PCR is considered as the gold standard for diagnosis, given that some clinical observations reported that the positive detection rate was only $30 \%-60 \%,{ }^{16}$ real-time PCR is inadequate for testing tear .samples ${ }^{17}$ Thus, in our study, only one-step RT-ddPCR successfully detected the virus in tears, suggesting that it is highly sensitive and is a feasible tool for the detection of the viral load in tears. In particular, considering that the tear sample is greatly diluted by the virus preservation solution, the low concentration of the virus in the preservation solution does not imply a low viral load in tears.

We analyzed the risk factors of tear positive patients and found no significant correlation with age, gender, disease severity, and CT findings. Interestingly, we found a significant correlation between tears and nasopharynxpositive patients but no correlation between tears and bloodpositive patients. We speculate that this may be owing to the expression of SARS-CoV-2-related receptors (ACE2 and CD147) in both the respiratory tract and ocular surface; this makes the virus more easily enriched in these two parts. ${ }^{18-22}$ Second, to the best of our knowledge, the ocular surface could connect with the respiratory tract through the nasolacrimal duct, and mucosal immune properties permitted the eye to be both a potential virus infected site as well as a gateway for respiratory infection. ${ }^{23}$ Notably, although animal experiments have confirmed that the SARS-CoV-2 can be inoculated through the eyes, it is still necessary to isolate the virus in the eyes to further prove that the virus can be transmitted through the eyes.

In our study, most triple-positive patients were sampled within 9 days after the illness onset, and most dual-positive patients were sampled within 20 days after the illness onset. A previous study reported that the SARS-CoV-2 viral load in throat swab and sputum samples was significantly correlated with the course of COVID-19: these viral loads peaked around 5-6 days after the onset of symptoms and gradually decreased until they could not be detected. ${ }^{24}$ Our findings agree with this dynamic law of SARS-CoV-2: the earlier the course of COVID-19 is, the higher the positive rate is, indicating a wider viral distribution and a higher viral load at the early stage. In fact, most of the cases were sampled over 1 week after the onset of symptoms, which means we might not catch the time window with the highest viral load.

Although our research has some important findings, there are still some limitations. First, as mentioned previously, our study did not enroll the early-stage COVID-19 patients who mainly remained in the community. For this reason, this study could not reflect the viral load in tears at the very early stage of COVID-19. Second, owing to the low incidence rate of conjunctivitis as well as the sharp decline of newly diagnosed cases, this study could not investigate the viral load in COVID-19 patients with conjunctivitis. Thus, further studies are better to investigate the viral load from the very early stage as well as in coexisting conjunctivitis patients.

In conclusion, by using one-step RT-ddPCR, our study demonstrates that the positive rate of SARS-CoV-2 in tears is greatly underestimated. Due to the dilution effect by the virus preservation solution, the low concentration of the virus in the preservation solution may not imply a low viral load in tears. Our study shows that the virus has a molecular basis for ocular transmission, but further study is needed to investigate its infectivity through eyes.

\section{Author Contributions}

All authors made a significant contribution to the work reported, whether that is in the conception, study design, execution, acquisition of data, analysis and interpretation, or in all these areas; took part in drafting, revising or critically reviewing the article; gave final approval of the version to be published; have agreed on the journal to which the article has been submitted; and agree to be accountable for all aspects of the work.

\section{Funding}

This study was funded by grants from the National Natural Science Foundation of P.R. China (Grant No. 81974136) and grants from the Huazhong University of science and Technology Foundation of P.R. China (Grant No. 2020kfyXGYJ068). The funding source had no role in the design and conduct of the study; collection, management, analysis, and interpretation of the data; preparation, review, or approval of the manuscript; and decision to submit the manuscript for publication.

\section{Disclosure}

The authors declare no competing interests.

\section{References}

1. COVID-19 weekly epidemiological update World Health Organization; November 3, 2020. Available from: https://www.who. $\mathrm{int} /$ publications $/ \mathrm{m} / \mathrm{item} /$ weekly-epidemiological-update-3-november2020. Accessed December 1, 2021. 
2. Gorbalenya AE, Baker SC, Baric RS, et al. Severe acute respiratory syndrome-related coronavirus: the species and its viruses a statement of the coronavirus study group. bioRxiv Preprint. 2020. doi: $10.1101 / 2020.02 .07 .937862$

3. World Health Organization. Coronavirus; 2020. Available from: https://www.who.int/health-topics/coronavirus. Accessed December $1,2021$.

4. Chen Y, Chen L, Deng Q, et al. The presence of SARS-CoV-2 RNA in feces of COVID-19 patients. J Med Virol. 2020;3:833-840. doi:10.1002/jmv.25825

5. Zhang X, Chen X, Chen L, et al. The evidence of SARS-CoV-2 infection on ocular surface. Ocul Surf. 2020;18(3):360-362. doi:10.1016/j.jtos.2020.03.010

6. Seah IYJ, Anderson DE, Kang AEZ, et al. Assessing viral shedding and infectivity of tears in coronavirus disease 2019 (COVID-19) patients. Ophthalmology. 2020;127:977. doi:10.1016/j. ophtha.2020.03.026

7. Xia J, Tong J, Liu M, Shen Y, Guo D. Evaluation of coronavirus in tears and conjunctival secretions of patients with SARS-CoV-2 infection. J Med Virol. 2020;26:589-594. doi:10.1002/jmv.25725.

8. Zhou YY, Zeng YY, Tong YQ, Chen CZ. Ophthalmologic evidence against the interpersonal transmission of 2019 novel coronavirus through conjunctiva. medRxiv Preprint. 2020;2:929-936. doi:10.1101/2020.02.11.20021956.

9. Mahmoud H, Ammar H, El Rashidy A, et al. Coronavirus in the conjunctival tears and secretions in patients with SARS-CoV-2 infection in Sohag Province, Egypt. Clin Ophthalmol. 2020;14:2701-2708. doi:10.2147/OPTH.S270006

10. Hindson CM, Chevillet JR, Briggs HA, et al. Absolute quantification by droplet digital PCR versus analog real-time PCR. Nat Methods. 2013;10(10):1003-1005. doi:10.1038/nmeth.2633

11. Yu F, Yan L, Wang N, et al. Quantitative detection and viral load analysis of SARS-CoV-2 in infected patients. Clin Infect Dis. 2020;28:ciaa345. doi:10.1093/cid/ciaa345

12. Racki N, Morisset D, Gutierrez-Aguirre I, Ravnikar M. One-step RT-droplet digital PCR: a breakthrough in the quantification of waterborne RNA viruses. Anal Bioanal Chem. 2014;406(3):661-667. doi:10.1007/s00216-013-7476-y

13. Chen L, Wang G, Long X, et al. Dynamics of blood viral load is strongly associated with clinical outcomes in COVID-19 patients: a prospective cohort study. J Mol Diagn. 2020;23(1):10-18.
14. World Health Organization. Clinical management of severe acute respiratory infection when novel coronavirus $(\mathrm{nCoV})$ infection is suspected: interim guidance; 2020. WHO/2019-nCoV/clinical/ 2020.5 .

15. Li Q, Guan X, Wu P, et al. Early transmission dynamics in Wuhan, China, of novel coronavirus-infected pneumonia. $N$ Engl J Med. 2020;382(13):1199-1207. doi:10.1056/NEJMoa2001316

16. Wang W, Xu Y, Gao R, et al. Detection of SARS-CoV-2 in different types of clinical specimens. JAMA. 2020;323(18):1843-1844. doi:10.1001/jama.2020.3786

17. Scherz W, Doane MG, Dohlman CH. Tear volume in normal eyes and keratoconjunctivitis sicca. Albrecht Von Graefes Arch Klin Exp Ophthalmol. 1974;192(2):141-150. doi:10.1007/BF00410700

18. Sun Y, Pan X, Liu L, Ni RX. Expression of SARS coronavirus $\mathrm{S}$ protein functional receptor ACE2 in human and rabbit cornea and conjunctiva. Rec Adv Ophthalmol. 2004;24(5):332-336.

19. Wang Q, Zhang Y, Wu L, et al. Structural and functional basis of SARS-CoV-2 entry by using human ACE2. Cell. 2020;181(4):894904.e9. doi:10.1016/j.cell.2020.03.045

20. Wang K, Chen W, Zhou YS, et al. SARS-CoV-2 invades host cells via a novel route: CD147-spike protein. bioRxiv Preprint. 2020. doi:10.1101/2020.03.14.988345

21. Määttä M, Tervahartiala T, Kaarniranta K, et al. Immunolocalization of EMMPRIN (CD147) in the human eye and detection of soluble form of EMMPRIN in ocular fluids. Curr Eye Res. 2006;31 (11):917-924. doi:10.1080/02713680600932290

22. Gheblawi M, Wang K, Viveiros A, et al. Angiotensin converting enzyme 2: SARS-CoV-2 receptor and regulator of the renin-angiotensin system. Circ Res. 2020;126(10):1456-1474. doi:10.1161/CIRCRESAHA.120.317015

23. Belser JA, Rota PA, Tumpey TM. Ocular tropism of respiratory viruses. Microbiol Mol Biol Rev. 2013;77(1):144-156. doi:10.1128/ MMBR.00058-12

24. Pan Y, Zhang D, Yang P, Poon LLM, Wang Q. Viral load of SARS-CoV-2 in clinical samples. Lancet Infect Dis. 2020;20 (4):411-412. doi:10.1016/S1473-3099(20)30113-4
Infection and Drug Resistance

\section{Publish your work in this journal}

Infection and Drug Resistance is an international, peer-reviewed openaccess journal that focuses on the optimal treatment of infection (bacterial, fungal and viral) and the development and institution of preventive strategies to minimize the development and spread of resistance. The journal is specifically concerned with the epidemiology of antibiotic resistance and the mechanisms of resistance development and diffusion in both hospitals and the community. The manuscript management system is completely online and includes a very quick and fair peerreview system, which is all easy to use. Visit http://www.dovepress.com/ testimonials.php to read real quotes from published authors. 